\title{
Bone cement with screw augmentation technique for the management of moderate tibial bone defects in primary knee arthroplasty patients with high body mass index
}

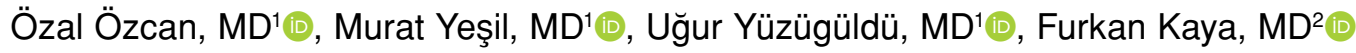 \\ ${ }^{1}$ Department of Orthopaedics and Traumatology, Faculty of Medicine, Afyonkarahisar Health Sciences University, Afyonkarahisar, Turkey \\ ${ }^{2}$ Department of Radiology, Faculty of Medicine, Afyonkarahisar Health Sciences University, Afyonkarahisar, Turkey
}

There is a certain consensus that total knee arthroplasty (TKA) surgery is an effective treatment option for patients with end-stage osteoarthritis of the knee. ${ }^{[1]}$ Although the success rate is usually high in TKA, there can be various occasional problems negatively influencing clinical outcomes. Definitely, tibial bone defects can be considered one of the most challenging issues that are encountered during TKA. It is known that bone defects can jeopardize implant durability, support or stabilization and create a significant challenge to surgeons in primary TKA. ${ }^{[1,2]}$ These bone defects can impact proper alignment of implants and eventually the extremities, if not properly treated. Management options for bone defects in primary TKA include bone grafts (autografts or allografts), ${ }^{[3]}$ bone cement, bone cement with screw reinforcement, ${ }^{[4]}$

Received: May 23, 2020

Accepted: September 10, 2020

Published online: January 06, 2021

Correspondence: Murat Yeşil, MD. Afyonkarahisar Sağlık Bilimleri Üniversitesi Tıp Fakültesi, Ortopedi ve Travmatoloji Anabilim Dalı, 03030 Afyonkarahisar, Türkiye.

E-mail: drmurat17@hotmail.com

Doi: $10.5606 /$ ehc. 2021.76491

Citation: Özcan Ö, Yeşil M, Yüzügüldü U, Kaya F. Bone cement with screw augmentation technique for the management of moderate tibial bone defects in primary knee arthroplasty patients with high body mass index. Jt Dis Relat Surg 2021;32(1):28-34.

\section{(C2021 All right reserved by the Turkish Joint Diseases Foundation}

This is an open access article under the terms of the Creative Commons Attribution-NonCommercial License, which permits use, distribution and reproduction in any medium, provided the original work is properly cited and is not used for commercial purposes (http://creativecommons.org/licenses/by-nc/4.0/).

\section{ABSTRACT}

Objectives: This study aims to investigate whether variables such as body mass index (BMI), size of the cement with screw augmentation area (CSA), distance between the base of tibial plate and the deepest point of the defect area (DPDA) may cause any mechanical problems leading to deterioration in tibiofemoral alignment or impact clinical outcomes when the surgeon utilizes bone cement with screw augmentation (BCSA) technique in the treatment of moderate non-contained tibial bone defects in total knee arthroplasty (TKA).

Patients and methods: This cross-sectional study, conducted between March 2018 and March 2019, included 37 knees of 28 patients (4 males, 24 females; mean age 71.3 \pm 8.9 ; range, 55 to 86 years) with moderate tibial bone defects requiring treatment with BCSA during primary TKA. Patients with BMI $>30$ were scored with Hospital for Special Surgery (HSS) score for clinical outcomes; besides, CSA, DPDA, and tibiofemoral alignment were calculated on plain X-rays.

Results: Mean BMI was 34.1 \pm 5.7 (range, 24.9 to 45.9). Patients had a mean follow-up period of $44 \pm 13.9$ (range, 28 to 75 ) months. Mean postoperative CSA was $98.2 \pm 35.3$ (range, 42 to 180) $\mathrm{mm}^{2}$ and DPDA was $7.4 \pm 2.6$ (range, 3.5 to 12.9 ) $\mathrm{mm}$. Mean HSS score at last follow-up was $88.0 \pm 7.5$ (range, 71 to 97 ).

Conclusion: Bone cement with screw augmentation technique was associated with satisfactory clinical outcomes and tibiofemoral alignment was not significantly deviated in patients with high BMI. We determined that neither the depth of DPDA nor the size of CSA had any correlation with clinical outcomes.

Keywords: Arthroplasty, augmentation, body mass index, cement, knee, primary, screw, tibial bone defect.

metal augmentation, ${ }^{[5]}$ and structural allografts or tantalum.

The bone cement with screw augmentation (BCSA) technique was initially introduced by Freeman et al. ${ }^{[6]}$ for the management of tibial bone defects encountered during TKA before Ritter ${ }^{[4]}$ popularized the technique in 1986. The previous literature 


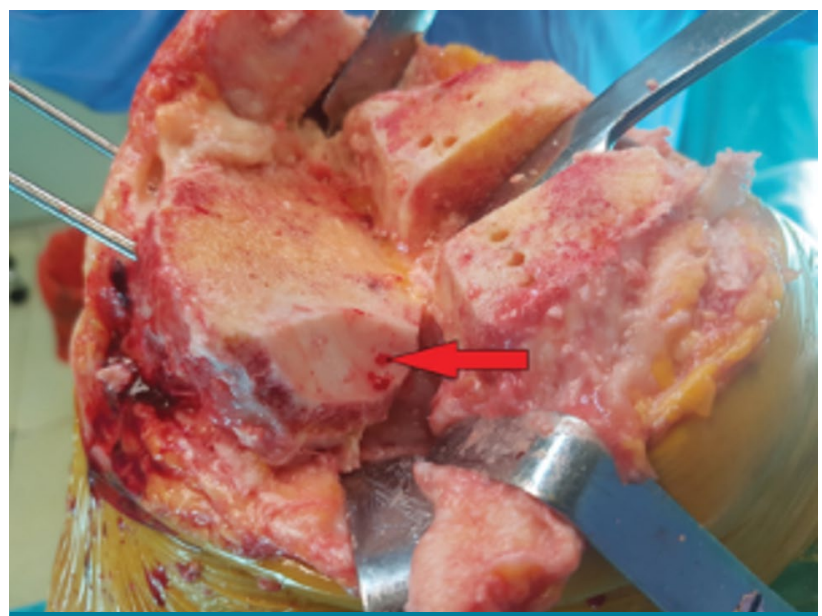

FIGURE 1. A non-contained tibial bone defect encountered during primary total knee arthroplasty (red arrow).

consists of various reports advocating the use of bone cement with screw in management for small tibial bone defects that do not extend beyond $5 \mathrm{~mm}$ in depth. ${ }^{[2,3,5]}$ There are also some additional reports about successful results with the cement and screw reinforcement technique in patients with relatively larger defects of the tibia that are up to $20 \mathrm{~mm}$ in the early or intermediate follow-up periods. ${ }^{[7]}$ $\operatorname{Ritter}^{[4]}$ also reported satisfactory outcomes for the management of relatively larger defects of the tibia (5 to $20 \mathrm{~mm}$ ). Yet, the existing literature remains inconsistent to date. ${ }^{[4,8,9]}$

Tibial bone defects can roughly be assigned to two major groups: contained or non-contained (Figure 1). Peripheral defects are usually located posteromedially in varus knees. ${ }^{[5]}$ The extent of bone loss in the tibia can be classified as follows: minimal, moderate, extensive, and massive cavitary (Table I). ${ }^{[5]}$ A moderate defect can be defined as having a depth of 5 to $10 \mathrm{~mm} \cdot{ }^{[5]}$ In this study, we aimed to investigate whether variables such as body mass index (BMI), size of the cement with screw augmentation area (CSA), distance between the base of tibial plate and the deepest point of the defect area (DPDA) may cause any mechanical problems leading to deterioration in tibiofemoral alignment or impact clinical outcomes when the surgeon utilizes BCSA technique in the treatment of moderate noncontained tibial bone defects in TKA.

\section{PATIENTS AND METHODS}

This cross-sectional study, conducted between March 2018 and March 2019, included patients who were identified from Department of Orthopaedics and Traumatology, Afyonkarahisar Health Sciences University arthroplasty database. We scanned our database and recruited patients with recorded moderate tibial bone defects encountered and managed during TKA. All patients had minimum two years of follow-up. Then, patients were invited to our outpatient clinic for further evaluation. Our database included 53 knees of 40 consecutively recorded patients with primary TKA treated for moderate tibial bone defects using the BCSA technique. After applying inclusion and exclusion criteria, 40 knees of 30 patients were identified as eligible for the study. Two patients were lost to follow-up and 37 knees of 28 patients (4 males, 24 females; mean age $71.3 \pm 8.9$; range, 55 to 86 years) with a minimum of 28 (mean $44 \pm 13.9$; range, 28 to 75 ) months of follow-up were included in statistical analysis. The study protocol was approved by the Afyonkarahisar Health Sciences University Ethics Committee (date: 02.03.2018, number: 2018/71). A written informed consent was obtained from each patient. The study was conducted in accordance with the principles of the Declaration of Helsinki.

Inclusion criteria were determined as being aged between 45 to 90 years with $\mathrm{BMI}>30$, having primary TKA, and treatment for tibial bone defects with the BCSA technique during TKA. Exclusion criteria were as follows: a history of revision knee arthroplasty,

\begin{tabular}{|lcc|}
\hline \multicolumn{4}{c}{ TABLE I } \\
\hline Type & Classification of tibial bone defects \\
\hline Minimal & Condylar involvement (\%) & Depth (mm) \\
Moderate & $<50$ & $<5$ \\
Extensive & $>50$ and $<70$ & $5-10$ \\
Cavitary & $>70$ and $<90$ & $\geq 10$ \\
(a) Intact peripheral rim & & \\
(b) Deficient peripheral rim & $>90$ & \\
\hline
\end{tabular}


valgus malalignment before surgery, primary or revision hip arthroplasty, fractures involving the lower extremity, intraoperative lateral release, amputation of lower extremities at any level, patients with tibial massive cavitary defects, tibial defects secondary to trauma, or pseudoarthrosis of the tibia.

All patients had undergone primary knee arthroplasty with Tipsan ${ }^{\circledR}$ Ps system $\left(\operatorname{Tipsan}^{\circledR}\right.$ A.Ş., Izmir, Turkey) in one or both knees by the same surgeon and BCSA technique was used in all cases for moderate tibial defects that were encountered during surgery (Figure 2). The BCSA technique was utilized in the same fashion as previously described by Ritter et al. ${ }^{[4]}$ We debrided the granulation tissue in the tibial defect area to expose sclerotic and cancellous bone. Then, we drilled multiple holes into the defective area with $2.7 \mathrm{~mm}$ drill bit and introduced $3.5 \mathrm{~mm}$ stainless steel cancellous screws (Figure 3). Afterwards, we injected cement into the drill holes, the cancellous and cortical area of the defect, and the entire area, respectively. The excess cement was shaved with a knife after the tibial plate was pressed into place.

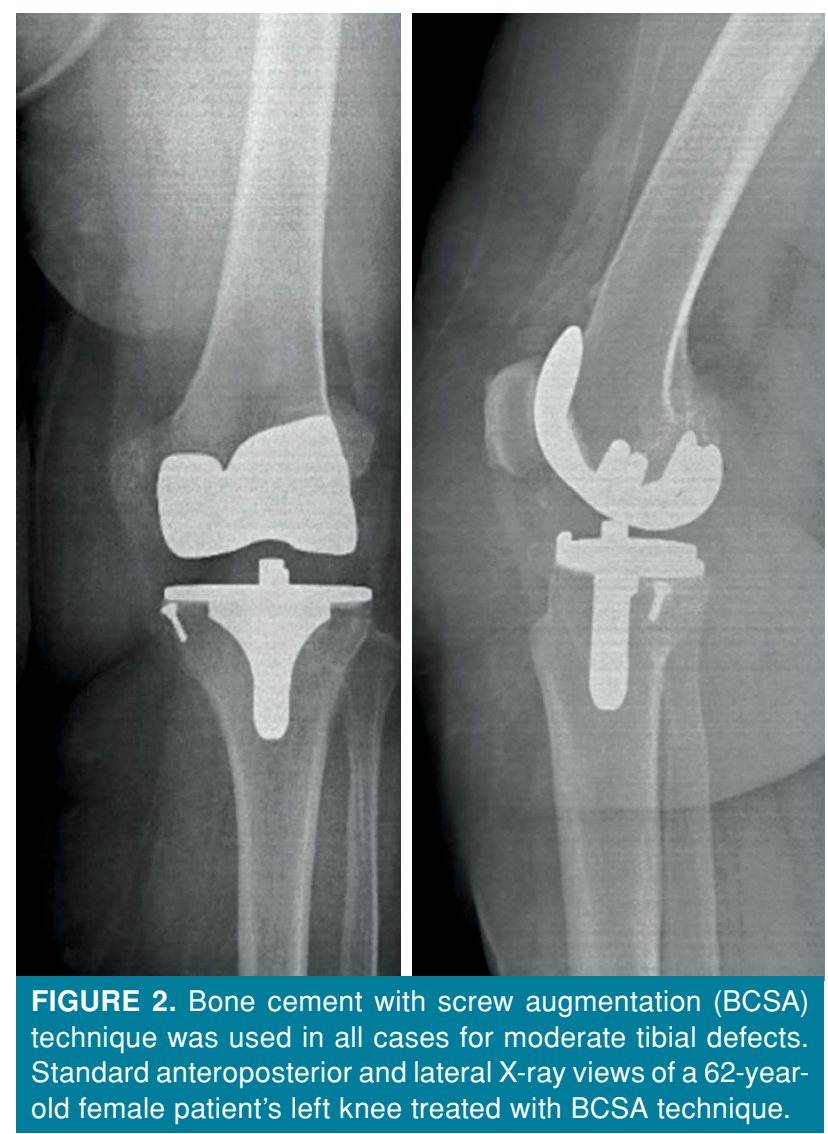

All patients were invited to our outpatient clinic for final clinical and radiologic evaluation. All demographic data were recorded along with BMI for each patient. All patients were scored by using the Hospital for Special Surgery (HSS) knee scoring system as the clinical outcome measure at final follow-up.

Plain radiographs in anteroposterior (AP) and lateral position (in $30^{\circ}$ of knee flexion) were obtained for each knee on the postoperative first day and at last follow-up visit. After obtaining X-ray images, early postoperative and latest AP radiographs of the knees were evaluated by the same radiologist. All measurements were carried out by the same radiologist using Picture Archiving and Communication System (PACS) (Enlil ${ }^{\oplus}$ Efe Eroğlu Bilişim Teknolojileri San. ve Tic. Ltd. Şti., Eskişehir, Turkey) software. Radiologic evaluation comprised tibiofemoral angle (TFA), fully-filled tibial defect area below the tibial plate, and the distance between the tibial plate and the deepest point in the fully-filled defect area.

Tibiofemoral angle was measured on routine AP knee X-ray images following the instructions described by Ishii et al. ${ }^{[10]}$ The femoral anatomical axis was drawn from the midpoint at the proximal end to a point $10 \mathrm{~cm}$ above the joint line and tibial anatomical axis was drawn from the midpoint at the proximal end to a point $10 \mathrm{~cm}$ above the joint line. These points were found to have the best correlation with the long X-ray images. ${ }^{[10]}$ Afterwards, TFA was measured between these two axes.

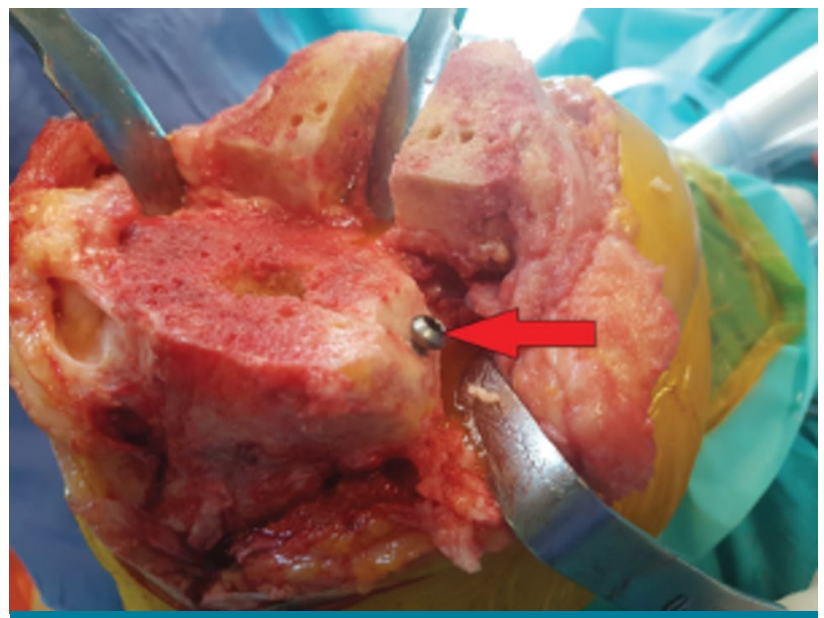

FIGURE 3. Defective area was drilled with $2.7 \mathrm{~mm}$ drill bit and introduced $3.5 \mathrm{~mm}$ stainless steel cancellous screws (red arrow). 


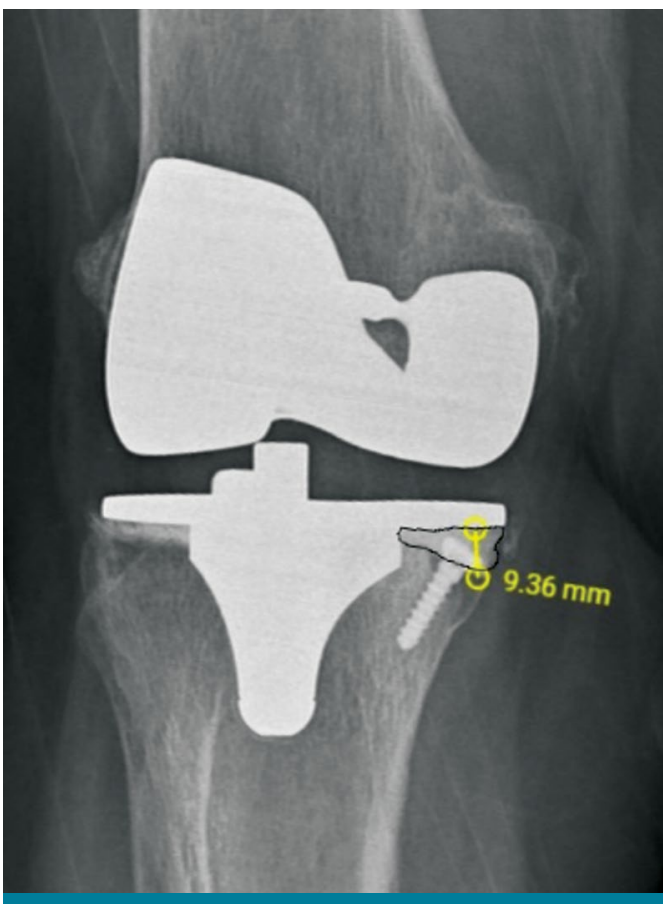

FIGURE 4. A 68-year-old female patient with a follow-up period of 45 months. Picture Archiving and Communication System software was used to measure size of cement with screw augmentation area (irregularly shaped bordered section below base plate) and distance between base of tibial plate and deepest point of defect area (line with two circles at each end).

In order to measure the fully-filled tibial defect area below the tibial plate, which will be referred to later in the text as CSA, PACS software was used by the same radiologist (Figure 4).

The distance between the base of tibial plate and the DPDA was measured using the digital ruler on PACS software by the same radiologist. In order to measure the distance, one parallel line was drawn to the base of the tibial plate and another perpendicular line was drawn to the deepest point of the tibial defect.

\section{Statistical analysis}

Statistical analysis was performed using the IBM SPSS version 20.0 software (IBM Corp., Armonk, NY, USA). Continuous variables were described using mean \pm standard deviation; categorical variables were described with proportions. Comparisons of pre- versus post-intervention values for continuous variables were performed using paired, two-sided Student's t-tests. Pearson correlation analyses were conducted to determine associations between the outcome parameters. A predetermined $p$ value of less than 0.05 was considered statistically significant.

\section{RESULTS}

Of the knees, $22(59.4 \%)$ were left and 15 (40.5\%) were right (Table II). According to measurements conducted on early postoperative AP radiographs of all 37 knees, nine $(24.3 \%)$ were in varus, 24 (64.9\%) were in valgus, and four $(10.8 \%)$ were in neutral position. Latest follow-up radiographs, obtained via inviting the patients our hospital, revealed that $12(32.4 \%)$ knees were in varus, $22(59.5 \%)$ were in valgus, and three $(8.1 \%)$ were in neutral position (Table III). Latest X-rays demonstrated that TFA of three patients was changed to varus position from their previous state of neutral or valgus position after the mean follow-up period of $44 \pm 13.9$ (range, 28 to 75 ) months.

Mean HSS score at last follow-up was $88.0 \pm 7.5$ (range, 71 to 97) (Table IV). Mean TFA on early postoperative radiographs was measured as $-2.27^{\circ} \pm 3.7^{\circ}$ (range, $-9.5^{\circ}$ to $4.7^{\circ}$ ) (in valgus), while

\begin{tabular}{|lccc|}
\multicolumn{4}{c|}{ TABLE II } \\
\\
\multicolumn{4}{|c|}{ Demographic data of patients } \\
\hline Age (year) & $\mathrm{n}$ & $\%$ & Mean \pm SD \\
Sex & 28 & 14.3 & $71.3 \pm 8.9$ \\
$\quad$ Female & & & \\
$\quad$ Male & 28 & 100 & \\
Body mass index (kg/cm $\left.{ }^{2}\right)$ & 24 & 85.7 & $34.1 \pm 5.7$ \\
Mean follow-up period & 28 & & $43.9 \pm 13.9$ \\
Total number of knees & 28 & & \\
$\quad$ Right knee & 37 & 100 & \\
$\quad$ Left knee & 15 & 40.5 & \\
\hline SD: Standard deviation. & 22 & 59.4 & \\
\hline
\end{tabular}




\begin{tabular}{|c|c|c|c|c|c|c|c|c|}
\hline \multicolumn{9}{|c|}{$\begin{array}{c}\text { TABLE III } \\
\text { Changes in tibiofemoral alignment }\end{array}$} \\
\hline & \multicolumn{2}{|c|}{ Valgus } & \multicolumn{2}{|c|}{ Neutral } & \multicolumn{2}{|c|}{ Varus } & \multirow[b]{2}{*}{ Mean $\pm S D$} & \multirow[b]{2}{*}{ Tota } \\
\hline & $\mathrm{n}$ & $\%$ & $\mathrm{n}$ & $\%$ & $n$ & $\%$ & & \\
\hline Early postoperative X-ray & 24 & 64.9 & 4 & 10.8 & 9 & 24.3 & $-2.27 \pm 3.7^{\circ}$ & 37 \\
\hline Late follow-up X-ray & 22 & 59.5 & 3 & 8.1 & 12 & 32.4 & $-1.69 \pm 3.7^{\circ}$ & 37 \\
\hline
\end{tabular}

latest follow-up radiographs demonstrated a mean TFA of $-1.69^{\circ} \pm 3.71^{\circ}$ (range, $-9.5^{\circ}$ to $+4.7^{\circ}$ ) (in valgus). There was no statistically significant difference between early postoperative and late radiographs regarding the TFA $(\mathrm{p}=0.115)$.

Mean postoperative CSA was $98.2 \pm 35.3$ (range, 42 to 180$) \mathrm{mm}^{2}$ and not correlated with early postoperative TFA $(\mathrm{p}=0.149)$ or late TFA $(\mathrm{p}=0.158)$ (Table IV).

Mean DPDA was $7.4 \pm 2.6$ (range, 3.5 to 12.9 ) $\mathrm{mm}$. DPDA was not correlated with age $(\mathrm{p}=0.896), \mathrm{BMI}$ $(\mathrm{p}=0.728)$ or HSS scores $(\mathrm{p}=0.603)$.

According to Pearson correlation test, HSS scores were not correlated with the defective area that was fully filled with CSA ( $p=0.597)$. Hospital for Special Surgery scores had negative correlation between early $(p=0.231)$ and late $(p=0.320)$ TFAs, although not statistically significant. Hospital for Special Surgery scores were strongly but not significantly correlated with late postoperative TFA $(\mathrm{p}=0.53)$.

Mean BMI of the patients was $34.1 \pm 5.7$ (range, 24.9 to 45.9) (Table II). Statistical analysis revealed that mean BMI of the patients was not correlated with HSS scores $(\mathrm{p}=0.082)$ or late postoperative TFA ( $p=0.591)$. Besides, no correlation was present between BMI and CSA $(p=0.261)$ or between BMI and changes in TFA ( $p=0.903)$.

During the follow-up period, we observed adverse TFA changes in three patients into varus position from neutral or valgus which we regarded

\begin{tabular}{|lcc|}
\hline \multicolumn{3}{|c}{ TABLE IV } \\
\multicolumn{1}{|c|}{ Clinical and radiologic evaluation } \\
\hline Hospital for Special Surgery Knee Score & 37 & $88.0 \pm 7.5$ \\
Deepest point of the defect area $\left(\mathrm{mm}^{2}\right)$ & 37 & $7.4 \pm 2.6$ \\
Cement with screw augmentation area $\left(\mathrm{mm}^{2}\right)$ & 37 & $98.2 \pm 35.3$ \\
\hline SD: Standard deviation. & & \\
\hline
\end{tabular}

as complications. However, there were no clinical complaints. Besides, no implant failure or need for revision was observed after the follow-up period.

\section{DISCUSSION}

We utilized the BCSA technique for the management of moderate tibial bone defects during TKA surgery and our patients had satisfactory clinical outcomes. Despite changes in numbers, statistical analysis did not demonstrate any significant deterioration in mean tibiofemoral alignment angle of the cases after the follow-up period. In addition, we observed that neither the amount of depth nor the size of the coronal plane area of moderate tibial bone defects had any correlation with clinical outcomes. Although the vast majority of patients had high BMI values exceeding 30, we did not observe any implant failure or major malalignment requiring revision surgery. Our results in a shorter follow-up period of 44 months were in line with those reported by Ritter ${ }^{[4]}$ and Ritter et al. $^{[8]}$ suggesting no failures with the BCSA technique in the long term.

Although it is known that tibial bone defects, up to a certain level of depth such as $<5 \mathrm{~mm}$, can be filled with cement solely, some authors advocate that filling tibial bone defects in this fashion will provide poor mechanical support for the implant. ${ }^{[1]}$ A potential drawback of the solo cement technique is that cement cannot be pressurized; therefore, laminations will form within the cement resulting in eventual shrinkage of the cement. ${ }^{[12]}$ We preferred to utilize BCSA technique for moderate tibial bone defects larger than $5 \mathrm{~mm}$ in depth.

A previous in vitro study by Brooks et al. ${ }^{[1]}$ suggested that filling tibial defects with the BCSA technique could create greater deflections in axial or varus loading than metal wedge or custom component techniques. However, there are various in vivo studies opposing these in vitro findings. In order to comment on this debate, we measured the TFA both after surgery and in the last follow-up visits. Our results showed deterioration in TFA to some degree; however, 
the mean amount of change was not statistically significant after the follow-up period. We did not observe any complaints or unsatisfactory clinical outcomes in the last appointment. Thus, we consider the BCSA technique relatively safe in terms of TFA failure in a period after 44 months.

A study by Ritter et al. ${ }^{[8]}$ included 57 knees with tibial defects varying from 4 to $13 \mathrm{~mm}$ and utilized the BCSA technique during primary TKA with no failures in long-term clinical follow-up of 13 years. In 1991, Lotke et al. ${ }^{[7]}$ reported that they had only two failures in a population of 59 patients with the BCSA technique; moreover, they reported that the BCSA technique could be implemented for tibial defects varying from 10 to $20 \mathrm{~mm}$ in depth with satisfactory outcome. Our results suggest that the mean distance between the base of tibial plate and the DPDA is not correlated with HSS scores. Besides, our patients had a mean HSS score of 88.0 (range, 71 to 97) which reflects satisfactory clinical outcomes. In other words, we suggest that the depth of the tibial defect has no negative effect while using the BCSA technique for moderate tibial defects.

We also investigated whether there was any correlation between BMI and clinical outcomes. To the best of our knowledge, our study is the only one examining the effect of high body mass index (BMI) on clinical outcomes after using the BCSA technique. Body mass index can be considered one of the most challenging factors in terms of success for this technique because it is a direct measure of body weight that has an obvious mechanical impact on the augmented defect area. With this study, we determined that BMI does not significantly affect the clinical outcomes or alignment of the extremities in the follow-up period. We treated patients with high BMI values exceeding 30, even up to 45.9 in a particular case. Despite high BMI values of the patients, we did not observe any implant failures or need for revision surgery after the follow-up period. Our results suggest that orthopedic surgeons may consider utilizing BCSA in patients with higher BMI.

Another matter of debate about the BCSA technique is whether the size of the defect area, which has been filled with cement, affects the clinical outcomes. A comprehensive review of the literature revealed that our study is the first investigating this question. A common expectation may be that the size of the treated area could be negatively correlated with clinical success; however, our results suggest no correlation between cement and augmented screw area (CSA) and HSS scores $(\mathrm{p}=0.597)$.
There are various cutting edge techniques suggested for the treatment of tibial bone defects during TKA. ${ }^{[13]}$ However, we experience that these implants are usually expensive and cannot be easily accessible on occasion. On the contrary, BCSA is considered as an easy-to-use and affordable technique. ${ }^{[14]}$ Besides, BCSA technique can assure relatively larger amount of bone stock for possible revision surgery in the future. We know that inadequate bone stock is a challenging problem for revision knee arthroplasty. ${ }^{[14]}$

There are some limitations of our study such as the low number of cases and lack of comparable groups treated with other new techniques. Also, the mean follow-up period was relatively shorter than other imminent studies in the literature. However, there are also some strong points of the study. For instance, our study is the first to simultaneously investigate clinical outcomes, radiologic data, and the influence of body weight after BCSA technique in primary knee arthroplasty. Also, all patients were operated by the same surgeon using the same surgical technique.

In conclusion, we think BCSA is a feasible technique for the treatment of moderate tibial defects encountered during TKA. It is safe, easy to perform, and affordable. In addition, the BCSA technique should be considered as a reliable alternative to other cutting edge but expensive options such as tantalum, metal augmentation or structural allografts in cases where surgeons have limited access to these. However, future randomized studies with larger populations and longer follow-up periods should be conducted for further investigation of the clinical success of the BCSA technique.

\section{Declaration of conflicting interests}

The authors declared no conflicts of interest with respect to the authorship and/or publication of this article.

\section{Funding}

The authors received no financial support for the research and/or authorship of this article.

\section{REFERENCES}

1. Lacko M, Jarčuška P, Schreierova D, Lacková A, Gharaibeh A. Tranexamic acid decreases the risk of revision for acute and delayed periprosthetic joint infection after total knee replacement. Jt Dis Relat Surg 2020;31:8-13.

2. Berend ME, Ritter MA, Keating EM, Jackson MD, Davis KE. Use of screws and cement in primary TKA with up to 20 years follow-up. J Arthroplasty 2014;29:1207-10.

3. Qiu YY, Yan CH, Chiu KY, Ng FY. Review article: Treatments for bone loss in revision total knee arthroplasty. J Orthop Surg (Hong Kong) 2012;20:78-86.

4. Ritter MA. Screw and cement fixation of large defects in total knee arthroplasty. J Arthroplasty 1986;1:125-9. 
5. Rand JA. Bone deficiency in total knee arthroplasty. Use of metal wedge augmentation. Clin Orthop Relat Res 1991;271:63-71.

6. Freeman MA, Bradley GW, Revell PA. Observations upon the interface between bone and polymethylmethacrylate cement. J Bone Joint Surg [Br] 1982;64:489-93.

7. Lotke PA, Wong RY, Ecker ML. The use of methylmethacrylate in primary total knee replacements with large tibial defects. Clin Orthop Relat Res 1991;270:288-94.

8. Ritter MA, Keating EM, Faris PM. Screw and cement fixation of large defects in total knee arthroplasty. A sequel. J Arthroplasty 1993;8:63-5.

9. Ritter MA, Harty LD. Medial screws and cement: a possible mechanical augmentation in total knee arthroplasty. J Arthroplasty 2004;19:587-9.

10. Ishii Y, Ohmori G, Bechtold JE, Sherman RE, Gustilo RB. Accuracy of the short radiograph in the measurement of the tibiofemoral angle. The Knee 1995;2:81-4.

11. Brooks PJ, Walker PS, Scott RD. Tibial component fixation in deficient tibial bone stock. Clin Orthop Relat Res 1984;(184):302-8.

12. Windsor RE, Insall JN, Sculco TP. Bone grafting of tibial defects in primary and revision total knee arthroplasty. Clin Orthop Relat Res 1986;205:132-7.

13. Angerame MR, Jennings JM, Holst DC, Dennis DA. Management of bone defects in revision total knee arthroplasty with use of a stepped, porous-coated metaphyseal sleeve. JBJS Essent Surg Tech 2019;9:e14.

14. Atik OŞ. Is there something new and interesting in my article? Eklem Hastalik Cerrahisi 2019;30:69.

15. Lombardi AV Jr, MacDonald SJ, Lewallen DG, Fehring TK. Four challenges in revision total knee arthroplasty: exposure, safe and effective component removal, bone deficit management, and fixation. Instr Course Lect 2019;68:217-30. 